\title{
Anomalous Concentration Effects on Phase Behavior and Nematic Order in Mixtures of Side-Chain Liquid Crystal Polymers and Low- Molecular-Weight Liquid Crystals
}

\author{
Bilin Zhuang and Zhen-Gang Wang* \\ Division of Chemistry and Chemical Engineering, California Institute of Technology, Pasadena, California 91125, United States
}

ABSTRACT: We study the anomalous concentration effects in mixtures of side-chain liquid crystalline polymers (SCLCPs) and low-molecular-weight liquid crystals (LMWLCs) by modifying the theory of Brochard, Jouffroy, and Levinson (BJL) to include the effects of the polymer backbone. Our new theory considers both the interaction of the polymer backbone with the global nematic field, as well as the local steric constraints due to the grafting of the side groups onto the polymer backbone. In addition, we consider the enhancement in the coupling between the pedant mesogens and the LMWLC molecules as the SCLCP concentration increases. The resulting phase diagram and nematic order parameters are in qualitative agreement with experimental observations, but drastically different from the predictions by the BJL theory.
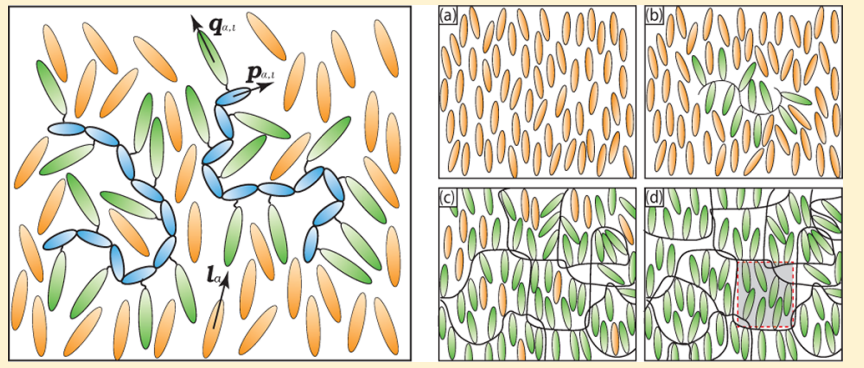

\section{INTRODUCTION}

Polymers and liquid crystals (LCs) are two broad classes of important materials. Incorporating rigid LC groups into polymeric structures results in liquid crystalline polymers that combine the ordering behavior of conventional LCs with the conformational degrees of freedom of polymers. ${ }^{1-5}$ Liquid crystalline polymers can be classified into three categories: the main-chain liquid crystalline polymer (MCLCP) consisting of a linear sequence of mesogenic LC segments, ${ }^{6}$ the side-chain liquid crystalline polymer (SCLCP), in which mesogenic LC segments are grafted onto the polymer backbone as pendant groups, ${ }^{7}$ and the combined main-chain/side-chain liquid crystalline polymer (MCSCLCP), which contains LCs on both the backbone and side groups. ${ }^{8}$ The SCLCPs can be further described as end-on or side-on depending on whether the mesogenic side groups are attached terminally ${ }^{9}$ or laterally ${ }^{10}$ onto the polymer backbone. In recent years, there is considerable interest in mixtures of liquid crystalline polymers and low-molecular-weight liquid crystals (LMWLCs) aimed at optimizing their thermal, mechanical and optical properties. ${ }^{5,11}$ In this work, we investigate the phase behavior and the nematic order of binary mixtures of SCLCPs and LMWLCs, with special attention to the effects of the polymer backbone on the phase transition and the nematic order parameters.

In mixtures of SCLCPs and LMWLCs, the interplay between the conformation of the polymer backbone and the liquid crystalline order generally results in anisotropic chain conformation. ${ }^{12}$ In the past 20 years, there have been many studies to determine the conformation of SCLCPs in nematic solvents using viscoelastic measurements ${ }^{13,14}$ or scattering experiments. $^{12,15,16}$ Early X-ray scattering experiments by Mattoussi et al. suggested that the conformation of end-on
SCLCP is slightly prolate in a nematic solvent. ${ }^{12,15}$ Yao et al., using viscoelastic measurements with analysis based on Brochard's hydrodynamic model, ${ }^{17}$ reported that the conformation of the end-on SCLCP in a nematic solvent changes from prolate to oblate as the molecular weight of SCLCP or the side-chain density increases or as the spacer length decreases. ${ }^{13}$ In recent years, using neutron scattering experiments, the Kornfield group reported that the conformation of the side-on SCLCP is highly prolate in nematic solvents, while that of the end-on SCLCP is slightly oblate. ${ }^{16,18}$ These experimental evidence on anisotropic chain conformation in SCLCPs clearly points to the coupling between the polymer backbone and the nematic order of the mesogen groups and can influence the liquid crystalline order of the side-chain and the solvent mesogens. ${ }^{19}$

In previous theoretical work on SCLCP/LMWLC mixtures, a common strategy, first proposed by Brochard, Jouffroy, and Levinson (hereafter referred to as BJL), ${ }^{20}$ and later extended by Kyu and co-workers, ${ }^{21,22}$ was to employ a phenomenological model that combines the Flory-Huggins theory of mixing ${ }^{23,24}$ and the Maier-Saupe theory of nematic order. ${ }^{25-27}$ While this approach can yield good agreement with the observed phase diagrams for some systems, ${ }^{22,28,29}$ it does not consider the conformation of the SCLCP backbone and any effects originating from the coupling between the polymer backbone and the nematic order. ${ }^{30,31}$ Another popular approach, proposed by Wang and Warner, ${ }^{32}$ describes the SCLCP backbone using a worm-like chain model and the coupling

Received: March 30, 2012

Revised: May 30, 2012

Published: July 18, 2012 
between the backbone and side groups using a term of the Maier-Saupe form. However, this theory predicts first-order phase transitions between nematic phases with different chain conformations, but such abrupt transitions have not been observed experimentally. ${ }^{4,33}$ A key shortcoming of the model is that it does not distinguish between the coupling of the SCLCP backbone with the global nematic order director and that with the locally grafted side groups. Recently, Wang and Wang ${ }^{31}$ developed a freely jointed chain model for SCLCP melts by considering both the global nematic interactions and the local couplings between the side-group and the backbone. This new model distinguishes between the side-on and the end-on SCLCPs through the sign of the local coupling parameter, and predicts that the chain conformation of the side-on SCLCP is always prolate, while that for the end-on SCLCP can be either prolate or oblate. In this work, we extend the model of ref 31 . for SCLCP melts to SCLCP/LMWLC mixtures, in order to address the interplay between chain conformation and liquid crystalline order for SCLCPs in nematic solvents.

In addition to the aforementioned coupling between the chain conformation and the liquid crystalline order, the attachment of mesogenic monomers to a polymer backbone, which restricts the freedom of the mesogenic groups, may also lead to enhanced nematic ordering. ${ }^{19,34,35}$ Earlier experiments suggested that the nematic phase is stabilized with increasing degree of polymerization of the SCLCP, ${ }^{19,34-39}$ possibly because of the denser packing of the mesogenic units due to their linkage to the polymer backbone. ${ }^{35}$ However, the consequences of this effect on the transition temperature and the nematic order in the SCLCP/LMWLC mixture have not been elucidated in previous literature. In solutions with a high concentration of SCLCPs, it is plausible that the LMWLC mesogens, dispersed in between SCLCPs, can also experience the packing effect caused by the polymer backbone, leading to an enhanced coupling between the side-group and the LMWLC mesogens as the polymer concentration increases. In this work, we account for this enhancement in the nematic interaction at high SCLCP concentration phenomenologically using a concentration dependent cross-interaction parameter between the side-group mesogens and the LMWLCs.

In a recent experiment, Scruggs and Kornfield studied the phase behavior and the nematic order parameters of SCLCP/ LMWLC mixtures in a series of measurements and found striking anomalous concentration effects. ${ }^{18,30}$ The SCLCP used in the experiment has a high degree of polymerization and a high grafting density, and the influence of the polymer backbone is expected to be important in this case. The observed relative nematic orders of the SCLCP side groups and the LMWLCs are contrary to the prediction by the BJL theory, which completely ignored the polymer backbone conformation. This experiment, to our knowledge, is the first work in which the nematic-to-isotropic transition temperature $T_{\mathrm{NI}}$ is measured in conjunction with the nematic order parameter for each of the mesogenic groups, and thus, it provides an excellent platform for testing and motivating theoretical models. In the present work, using the observations in the Scruggs-Kornfield experiment as a motivation, we explore the possible consequences of the polymer backbone on the phase transition and nematic order in SCLCP/LMWLC mixtures. However, the validity of our theory is not tied to this particular experiment.

The rest of this article is organized as follows. In section II, we describe the key experimental findings in the ScruggsKornfield experiment and note the discrepancy with predictions by the BJL model. In section III, we formulate our new theory by taking into account the coupling between the SCLCP backbone and the side groups, a key effect not considered in the BJL model. In section IV, we present the results from our calculation and compare to the experimental observations, and finally, in section $\mathrm{V}$, we offer some concluding remarks.

\section{MOTIVATING EXPERIMENTAL OBSERVATIONS}

Scruggs and Kornfield ${ }^{30}$ examined a series of SCLCP/LMWLC mixtures using $350 \mathrm{HSiCB} 4$ as the SCLCP and $5 \mathrm{CB}$ as the LMWLC. The chemical structures of both compounds are given in Figure 1. Henceforth, mesogenic groups refer to both

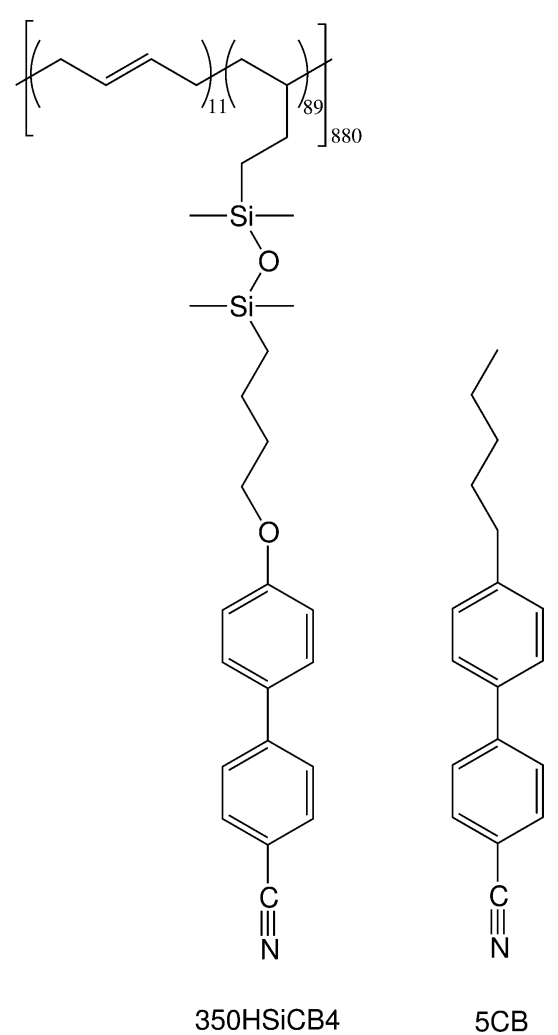

Figure 1. Chemical structures of the SCLCP $(350 \mathrm{HSiCB} 4)$ and the LMWLC (5CB).

the rigid SCLCP side groups and the LMWLCs, and the concentration $\phi$ refers to the volume fraction of the SCLCP in the mixture. They measured $T_{\mathrm{NI}}$ using differential scanning calorimetry, and the order parameters using deuterium NMR and refractometry. Compared to prior experiments on SCLCP/ LMWLC mixtures, ${ }^{22,28,40,41}$ the experiment by Scruggs and Kornfield used a much longer SCLCP chain with polymerization degree of 880 , and it is therefore expected that the influence of the SCLCP backbone is much more pronounced in their results.

There are three key observations in the Scruggs-Kornfield experiment that cannot be explained by the BJL theory, or by any other previous theories. First, the phase diagram (in temperature vs composition) is convex at low concentration but concave at high concentration. As $\phi$ changes from zero to one, $T_{\mathrm{NI}}$ first stays rather insensitive to concentration change for $\phi<0.2$, then increases to a maximum value of $78{ }^{\circ} \mathrm{C}$ at $\phi \approx$ 0.8 before decreasing to the $\mathrm{T}_{\mathrm{NI}}$ of the pure SCLCP at $63{ }^{\circ} \mathrm{C}$. The curvature of the phase diagram changes from convex to 
concave at around $\phi=0.5$. Contrary to the experimental observations, the BJL theory predicts a nematic-isotropic phase boundary that is either concave or convex in the entire concentration range.

Second, even though the nematic interaction between the SCLCP side groups is stronger than that between the LMWLCs, as suggested by the higher $\mathrm{T}_{\mathrm{NI}}$ for the pure SCLCP $\left(63{ }^{\circ} \mathrm{C}\right)$ than for the pure LMWLC $\left(35^{\circ} \mathrm{C}\right)$, the measured nematic order parameter of the SCLCP side groups is consistently lower than that of the LMWLC at concentrations below $10 \%$ weight of SCLCP. This behavior is opposite to that predicted by the BJL model.

Third, at low concentrations, the order parameter of the LMWLC decreases with increasing concentration, while the order of the SCLCP side groups is less sensitive to changes in concentration. In the BJL theory, however, the nematic orders of the two mesogenic groups show similar sensitivities to variation in concentration.

In the present work, we use these experimental observations as a guide to explore the effects of the polymer backbone on the phase transition and nematic order. As alluded to earlier, a major deficiency in the BJL theory is its neglect of the SCLCP backbone. Although the polymer backbone as a whole is flexible, the monomer units can be oriented by the pendant mesogen groups and LMWLC molecules, and through the local coupling to the pendant mesogen groups that are hinged to the backbone, can in turn influence the nematic order of the system. We include the coupling of SCLCP backbone with the mesogens using a model recently proposed in ref 31 . We show that the anomalous behavior in the solution properties mentioned in this section can be explained by a concentration dependent coupling between the pendant mesogens on the SCLCP and the LMWLC molecules. In section III, we present the details of our model, and in section IV, we discuss the results from our theory, and compare them with the predictions from the BJL model.

\section{THEORY}

Within the mean-field framework, we describe the SCLCP/ LMWLC mixture as a homogeneous binary solution of $n_{p}$ SCLCP chains and $n_{l}$ LMWLC mesogens. A schematic of the system is shown in Figure 2. We use the subscripts $p, q$, and $l$ to denote the SCLCP backbone segments, the SCLCP side groups, and the LMWLCs, respectively. The chain backbone of

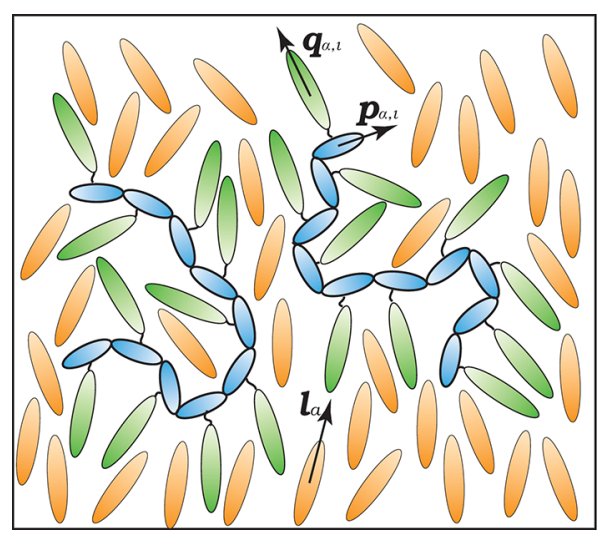

Figure 2. Schematic of the SCLCP/LMWLC mixture. The blue, green and orange segments represent the SCLCP backbone segments, the SCLCP side groups, and the LMWLCs, respectively. the SCLCP is modeled as a freely jointed chain of $N$ segments, each with a volume of $v_{p}$ and a well-defined long axis $\boldsymbol{p}_{\alpha, i}$, where $\alpha$ is the polymer chain index and $i$ is the segment index on each chain. An LC side-group is grafted onto each repeating segment of the SCLCP backbone through a flexible spacer. Each sidegroup has a volume of $v_{q}$ and a long axis $\boldsymbol{q}_{\alpha, i}$. For simplicity, the flexible spacer is not explicitly modeled; however, its volume contributes to a larger effective volume $v_{p}$ of the chain backbone segments. The LMWLCs are similarly described as monomers with volume $v_{l}$ and long axis $l_{j}$, where $j$ is the index for the LMWLC mesogens.

In the same spirit as the BJL model, we write the free energy of the system as a sum of the free energy of mixing and the Maier-Saupe free energy of nematic ordering of liquid crystals. Thus,

$$
f=f_{\text {mix }}+f_{\text {nem }}
$$

where $f_{\text {mix }}$ is the free energy density due to mixing and $f_{\text {nem }}$ is the free energy density due to nematic ordering.

We formulate the free energy of mixing using the FloryHuggins theory, to write by ${ }^{23,24}$

$$
\begin{aligned}
\frac{f_{\text {mix }}}{k_{\mathrm{B}} T}= & \frac{\phi}{N\left(v_{p}+v_{q}\right)} \log \phi+\frac{1-\phi}{v_{l}} \log (1-\phi) \\
& +\chi \phi(1-\phi)
\end{aligned}
$$

where $k_{B}$ is the Boltzmann constant, $T$ is the absolute temperature, and $\phi$ represents the volume fraction of the SCLCP given by

$$
\phi=\frac{n_{p} N\left(v_{p}+v_{q}\right)}{n_{p} N\left(v_{p}+v_{q}\right)+n_{l} v_{l}}
$$

$\chi$ is a phenomenological parameter that accounts for the effective interaction between the two components. Here, $\chi$ is defined without a reference volume, and thus, it has the dimension of $1 /$ volume. We take $\chi$ to be of the following form $^{24}$

$$
\chi=A+B / T
$$

reflecting both enthalpic and entropic origins of this effective interaction.

We model the nematic Hamiltonian of the system by extending the work in ref 31 for SCLCP melts, in which the free energy consists of interactions between the SCLCP backbone and the global nematic field, as well as the local steric constraints due to the side groups grafted onto the backbone. Also, while the BJL theory describes the nematic order of the system using only two order parameters respectively for the side groups and the LMWLC mesogens, the model in ref 31 has an additional order parameter that characterizes the order in the SCLCP backbone. To derive an expression for the nematic free energy, we start with a full nematic Hamiltonian that consists of both global nematic interactions and local hinging effects:

$$
\mathcal{H}=\mathcal{H}_{\text {global }}+\mathcal{H}_{\text {local }}
$$

The global-interaction term describes the collective nematic interaction at the level of the entire system. It is a molecular field approach that accounts for both the packing effects and the anisotropic energetic interactions. At this level, we use spatially dependent tensorial nematic order densities, $\boldsymbol{S}_{p}(\boldsymbol{r})$, $S_{q}(r)$, and $S_{l}(r)$ to describe the nematic order of the SCLCP 
backbone, the SCLCP side groups, and the LMWLCs, respectively. At the level of the Maier-Saupe theory, $\mathcal{H}_{\text {global }}$ is given by ${ }^{31}$

$$
\begin{aligned}
\mathcal{H}_{\text {global }} & {\left[\mathbf{S}_{p}(\mathbf{r}), \mathbf{S}_{q}(\mathbf{r}), \mathbf{S}_{l}(\mathbf{r})\right] } \\
& =-\frac{1}{2} U_{p p} \int \mathrm{d} \mathbf{r} \mathbf{S}_{p}(\mathbf{r}): \mathbf{S}_{p}(\mathbf{r})-\frac{1}{2} U_{q q} \int \mathrm{d} \mathbf{r} \mathbf{S}_{q}(\mathbf{r}): \mathbf{S}_{q}(\mathbf{r}) \\
& -\frac{1}{2} U_{l l} \int \mathrm{d} \mathbf{r} \mathbf{S}_{l}(\mathbf{r}): \mathbf{S}_{l}(\mathbf{r})-U_{p q} \int \mathrm{d} \mathbf{r} \mathbf{S}_{p}(\mathbf{r}): \mathbf{S}_{q}(\mathbf{r}) \\
& -U_{p l} \int \mathrm{d} \mathbf{r} \mathbf{S}_{p}(\mathbf{r}): \mathbf{S}_{l}(\mathbf{r})-U_{q l} \int \mathrm{d} \mathbf{r} \mathbf{S}_{q}(\mathbf{r}): \mathbf{S}_{l}(\mathbf{r})
\end{aligned}
$$

where $U_{k k^{\prime}}$ is a phenomenological parameter that characterizes the strength of nematic interactions between species $k$ and $k^{\prime}$ (with $k, k^{\prime}=p, q, l$ ). On the basis of physical consideration, all these parameters are positive since the mesogenic groups prefer parallel alignment; their magnitude depends on both the van der Waals forces and the steric influences. ${ }^{27}$ The nematic order parameters $\boldsymbol{S}_{k}(\boldsymbol{r})$ are related to the orientational vectors of the mesogens through:

$$
\begin{aligned}
& \mathbf{S}_{p}(\mathbf{r})=v_{p} \sum_{\alpha=1}^{n_{p}} \sum_{i=1}^{N} \delta\left(\mathbf{r}-\mathbf{r}_{\alpha, i}\right)\left(\mathbf{p}_{\alpha, i} \mathbf{p}_{\alpha, i}-\frac{1}{3} \mathbf{I}\right) \\
& \mathbf{S}_{q}(\mathbf{r})=v_{q} \sum_{\alpha=1}^{n_{p}} \sum_{i=1}^{N} \delta\left(\mathbf{r}-\mathbf{r}_{\alpha, i}\right)\left(\mathbf{q}_{\alpha, i} \mathbf{q}_{\alpha, i}-\frac{1}{3} \mathbf{I}\right) \\
& \mathbf{S}_{l}(\mathbf{r})=v_{l} \sum_{j=1}^{n_{s}} \delta\left(\mathbf{r}-\mathbf{r}_{j}\right)\left(\mathbf{l}_{j} \mathbf{l}_{j}-\frac{1}{3} \mathbf{I}\right)
\end{aligned}
$$

where $\boldsymbol{r}_{\alpha, i}$ and $\boldsymbol{r}_{j}$ are the position vectors of the labeled polymer segment and LMWLC molecule, respectively.

The local-interaction term, $\mathcal{H}_{\text {local }}$, describes the local coupling between a grafted side-group mesogen and the polymer backbone segment it is attached to. We model this local hinge effect by assuming that the energy is quadratic with the vector dot product between each pair of side groups and backbone segments: ${ }^{31}$

$$
\mathcal{H}_{\text {local }}=-u_{c} \sum_{\alpha=1}^{n_{p}} \sum_{i=1}^{N}\left(\mathbf{p}_{\alpha, i} \cdot \mathbf{q}_{\alpha, i}\right)^{2}
$$

Here $u_{c}$ is the local coupling parameter. The magnitude of $u_{c}$ depends on the rigidity of the grafting, and its sign determines whether parallel or perpendicular alignment between the sidegroup and the backbone is energetically favored. In this work, we consider end-on SCLCPs, in which the side-group mesogens are terminally grafted onto the polymer backbone, and therefore, $u_{c}$ is taken to be negative. A key difference between this local coupling term and the global interaction term is that the local coupling term exists (i.e., is nonvanishing) even in the isotropic phase, a crucial feature that was missed in earlier models for the hinge effect. ${ }^{32}$ Note that this local coupling term cannot be written in terms of the nematic order parameters. In addition, since the global interaction prefers a parallel alignment between the side-chain and the backbone, but the local coupling prefers a perpendicular one, singling out the effects of local grafting allows us to account for the actual magnitude of global packing more accurately.

An expression for the free energy density, $f_{\text {nem }}$ can be derived from the Hamiltonian by applying the self-consistent field theory. ${ }^{31,42-44}$ For a general, systematic derivation, we refer interested readers to the above-mentioned references. Basically, the derivation involves a series of identity transformations in the partition function using functional integration of field variables and then making the saddle-point approximation of the functional integration. In essence, the selfconsistent field approximation reduces an intractable manybody problem to an effective single-body problem in external fields which are then determined self-consistently. For spatially uniform systems with uniaxial order, which we take to be in the $z$-direction, the tensorial nematic order parameters are diagonal and traceless, and can be written using the scalar nematic order parameters $S_{k}$ as

$$
\mathbf{S}_{k}=\frac{S_{k}}{3}(-\hat{\mathbf{x}} \hat{x}-\hat{\mathbf{y}} \hat{y}+2 \hat{\mathbf{z}} \hat{z})
$$

Furthermore, with the freely jointed chain model for the polymer backbone, the partition function of the chain factorizes into product of the partition function for each repeating segment. The partition function for a repeating segment of the SCLCP, $Q_{p q}$, is given by

$$
\begin{aligned}
Q_{p q}= & \int \mathrm{d} \Omega_{p} \int \mathrm{d} \Omega_{q} \exp \left\{\beta v_{p}\left(u_{p p} \phi_{p} S_{p}+u_{p q} \phi_{q} S_{q}+u_{p l} \phi_{l} S_{l}\right)\right. \\
& P_{2}\left(\cos \theta_{p}\right)+\beta v_{q}\left(u_{p q} \phi_{p} S_{p}+u_{q q} \phi_{q} S_{q}+u_{q l} \phi_{l} S_{l}\right) P_{2} \\
& \left.\left(\cos \theta_{q}\right)+\beta u_{c}(\mathbf{p} \cdot \mathbf{q})^{2}\right\}
\end{aligned}
$$

and the partition function for the LMWLC solvent, $Q_{l}$, is

$$
Q_{l}=\int \mathrm{d} \Omega_{l} \exp \left\{\beta v_{l}\left(u_{p l} \phi_{p} S_{p}+u_{q l} \phi_{q} S_{q}+u_{l l} \phi_{l} S_{l}\right) P_{2}\left(\cos \theta_{l}\right)\right\}
$$

In these equations, $P_{2}(x)=\left(3 x^{2}-1\right) / 2$ is the second Legendre polynomial, $u_{k k^{\prime}}=2 / 3 U_{k k^{\prime}}$ is the reduced interaction parameter, and the integration is over the solid angle $\Omega_{k}$ for each of the mesogen species. The factor $2 / 3$ is introduced so that $u_{k k^{\prime}}$ match the parameters in the Maier-Saupe theory. The nematic order parameters, $S_{p}, S_{q}$, and $S_{v}$ are determined selfconsistently by taking the average with weights given by the Boltzmann factors in the integrands of eq 12 and eq 13:

$$
S_{k}=\left\langle P_{2}\left(\cos \theta_{k}\right)\right\rangle
$$

Equations 12, 13, and 14 constitute a complete set of selfconsistent equations that can be solved to obtain the nematic order parameters $S_{k}$. The nematic free energy density can be shown to be

$$
\begin{aligned}
f_{\text {nem }}= & \frac{F_{\text {nem }}}{V} \\
= & \frac{1}{2} u_{p p} \phi_{p}^{2} S_{p}^{2}+\frac{1}{2} u_{q q} \phi_{q}^{2} S_{q}{ }^{2}+\frac{1}{2} u_{l l} \phi_{l}{ }^{2} S_{l}^{2} \\
& +\frac{1}{2} u_{p q} \phi_{p} \phi_{q} S_{p} S_{q}+\frac{1}{2} u_{p l} \phi_{p} \phi_{l} S_{p} S_{l}+\frac{1}{2} u_{q l} \phi_{q} \phi_{l} S_{q} S_{l} \\
& -k T \frac{\phi}{v_{p}+v_{q}}\left(\ln Q_{p q}-\ln Q_{p q, 0}\right) \\
& -k T \frac{1-\phi}{v_{l}}\left(\ln Q_{l}-\ln 4 \pi\right)
\end{aligned}
$$

where $Q_{p q, 0}$ is the value of $Q_{p q}$ in the isotropic state given by

$$
Q_{p q, 0}=\int \mathrm{d} \Omega_{p} \int \mathrm{d} \Omega_{q} \exp \left[\beta u_{c}(\mathbf{p} \cdot \mathbf{q})^{2}\right]
$$


Note that this free energy is in reference to the isotropic state, so that for the isotropic phase $\left(S_{k}=0\right.$ for all $\left.k\right), f_{\text {nem }}=0$. Note also that the sign change in the interaction terms naturally arises from the derivation of the self-consistent field; in essence the energetic terms with the altered sign correct for the overcounting the energetic terms already included in the singlemolecule partition function. The free energy expression reduces to that in the BJL model when $u_{p p}, u_{p q}, u_{p l}$ and $u_{c}$ are zero.

At a fixed concentration, the nematic free energy $f_{\text {nem }}$ can be used to determine the relative stability between the isotropic phase and the nematic phase. However, the isotropic to nematic transition is often accompanied by a change in the composition, leading to two coexisting phases with different compositions. To determine the phase coexistence, we must equate the chemical potential for each species in the two coexisting phases. An equivalent, but mathematically more convenient approach is to work with the grand potential density $w$, defined as

$$
w=f-\mu \phi
$$

where $f$ is the Helmholtz free energy density given by eq 1 , and $\mu$ is a chemical-potential like variable conjugate to the polymer volume fraction $\phi$. It can be shown that the equality of chemical potential for each species is equivalent to

$$
\begin{aligned}
& \mu\left(\phi^{\mathrm{I}}\right)=\mu\left(\phi^{\mathrm{N}}\right) \\
& w\left(\phi^{\mathrm{I}}\right)=w\left(\phi^{\mathrm{N}}\right)
\end{aligned}
$$

where the superscripts I and $\mathrm{N}$ denote the isotropic phase and the nematic phase, respectively.

For the numerical calculations, it is convenient to reduce the parameters to dimensionless quantities. Since the pure LMWLC is modeled with the Maier-Saupe theory, we have $k T_{\mathrm{NI}, l} / u_{l l}=\alpha$, where $T_{\mathrm{NI}, l}$ is the isotropization temperature of pure LMWLC and $\alpha=4.5415$. Hence, a natural choice is to use $u_{l l}$ in defining the reduced parameters. We can replace the following quantities by their corresponding reduced dimensionless quantities indicated with a superscript $r$ :

$$
\begin{aligned}
& u_{k k^{\prime}}^{\mathrm{r}}=\frac{u_{k k^{\prime}}}{u_{l l}} \\
& u_{c}^{\mathrm{r}}=\frac{u_{c}}{u_{l l}} \\
& B^{\mathrm{r}}=\frac{B}{u_{l l}} \\
& \beta^{\mathrm{r}}=\beta u_{l l}
\end{aligned}
$$

In the subsequent text, we will work with reduced quantities and drop the superscript $r$. The equations remain the same with reduced quantities.

\section{RESULTS AND DISCUSSIONS}

In this section, we first explain the rationale for the choice of parameters used in our calculation in part A. Then, we present the phase diagrams and the nematic order parameters predicted by our new model and compare them with the results from the BJL model in parts B and C. Finally, in part D, we discuss the concentration dependence of the SCLCP chain conformation calculated from our model.

A. Choice of Parameters. In this part, we assign a reasonable set of parameters based on the geometric and physical consideration of the SCLCP/LMWLC mixture used in the Scruggs-Kornfield experiment. According to the chemical structure shown in Figure 1, the mesogens in both the SCLCP side-group and the LMWLC have the same volume and very similar structure, and therefore, we take the mesogen volume as a unit volume and set $v_{l}=v_{q}=1$. It is more difficult to determine the effective volume of the SCLCP backbone segments $v_{p}$. Even though the backbone does not consist of rigid rod-like segments, the crowding by the side-chains forces the backbone to have a much longer persistence length than the bare chain, ${ }^{45,46}$ and therefore, the backbone segments should have rod-like characters. We assign a value of $v_{p}=0.5$, so that the volume ratio of the SCLCP repeating unit to the LMWLC monomer is approximately equal to their weight ratio in the experiment. There is no explicit volume for the flexible spacer in our model; however, we consider that its effective volume is included into $v_{p}$.

The nematic interaction parameters $u_{k k^{\prime}}$ reflect the strength of anisotropic van der Waals interactions and steric repulsions. ${ }^{27,47}$ The value of $u_{l l}$ is set to 1 by default since the interaction parameters are scaled by $u_{l l}$ as in eq 20a. Other nematic interaction parameters are set relative to $u_{l l}$. Since $T_{\mathrm{NI}}$ of the SCLCP is $28{ }^{\circ} \mathrm{C}$ higher than that of the LMWLC, we assign $u_{q q}=1.7$ to account for the fact that the nematic interaction between SCLCP side groups is stronger than that between the LMWMC. This value of $u_{q q}$ is higher than the value required by the simple Maier-Saupe theory, because the local coupling effect included in our model frustrates the nematic ordering in the system and acts to lower the $T_{\mathrm{NI}}{ }^{31}$ The parameter for the local coupling is set to $u_{c}=-1$, describing a moderate hinging effect between the side-group and the backbone in the end-on SCLCP. On the other hand, the polymer backbone segments do not have an intrinsically rigid chemical structure, and we expect any global nematic interaction involving the polymer backbone to be weak. However, on account of the fact that flexible polymers can be oriented in a nematic solvent ${ }^{48}$ and that end-on SCLCPs can adopt a prolate conformation, ${ }^{12,15}$ there is generally a finite coupling between the polymer backbone and the mesogens. Furthermore, earlier theoretical work show that flexible spacer segments on MCLCPs may also develop partial order in the nematic phase. ${ }^{49-51}$ As such, we set $u_{p q}=u_{p l}=0.2$. For simplicity, we neglect the nematic coupling among polymer backbone segments, setting $u_{p p}=0$. Because of the weak nematic interactions of the backbone, the nematic ordering is driven by the liquid crystalline order in the side-group and the solvent and not by the polymer backbone.

The stronger nematic interaction between the SCLCP side groups than between the LMWLCs suggests that the presence of the polymer backbone can reinforce the nematic order, even though the local coupling between the backbone and the sidegroup has the opposite effect. ${ }^{19}$ Earlier experiments observed that the $T_{\mathrm{NI}}$ of the SCLCP increases with the degree of polymerization, ${ }^{19,34-39}$ and that the polymer backbone has a slight oblate conformation in the nematic phase, ${ }^{52-54}$ suggesting that the polymer backbone is aligned perpendicularly to the mesogens. At high grafting densities, such a configuration could give rise to local fluctuations of the smectic A character, as illustrated in the shaded region in Figure $3 \mathrm{~d}^{55-58}$ The reduction in the translational degrees of freedom of the mesogens-which is associated with the appearance of nematic order-is probably a consequence of the perpendicular grafting of the pendant mesogens to the polymer backbone. 

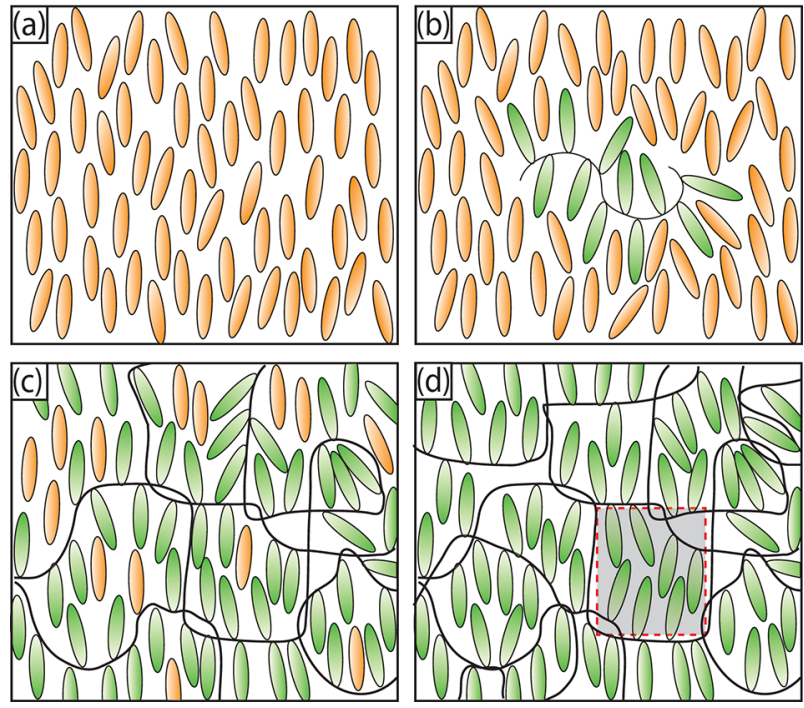

Figure 3. Schematic representation of SCLCP/LMWLC mixtures at various concentrations. The green and the orange segments represent LMWLCs and SCLCP side groups respectively, and the solid line represents the SCLCP backbone.The shaded region in part $d$ represents the local smectic fluctuation.

While the exact physical mechanism remains to be elucidated, it is possible that the polymer backbone and the spacers tend to be confined in layers between the aligned mesogenic side groups, as it is energetically unfavorable for the backbone to penetrate into the domains of aligned mesogens. ${ }^{59,60}$ This local layering effect confines both the rotational and translational motions of the mesogens in between, giving rise to local smectic fluctuations, and consequently contributing to a higher apparent global nematic interaction between the SCLCP side groups. We will henceforth refer to this effect as a confinement effect. We emphasize that we are not considering the smectic phase in this work; the local smectic fluctuations are only transient effects due to the confinement by the polymer backbone, whose treatment from a more fundamental theory is beyond the scope of this work.

The discussion of the enhanced nematic coupling due to confinement effect in the pure SCLCP has implications for the choice of the nematic cross-interaction parameter $u_{q l}$ between the SCLCP side-group and the LMWLC. In previous studies that apply the BJL model to SCLCP/LMWLC mixtures, $u_{q l}$ is assumed to be concentration-independent. ${ }^{20-22,61-63}$ In particular, Chiu and Kyu proposed that $u_{q l}=c\left(u_{q q} u_{l l}\right)^{1 / 2}$, where $c$ is a proportionality constant that characterizes the relative magnitude of the cross-interaction parameter between dissimilar mesogens to the geometric mean of the interaction parameters within the pure mesogens. ${ }^{21}$ The nematic order is destabilized by mixing if $c<1$, and stabilized if $c>1$. However, based on the experimental observations described in section II, such a form is insufficient to capture the phase behavior and the nematic orders of the mixtures. We propose to account for the confinement effect due to the polymer backbone by making the cross-nematic interaction dependent on the concentration. Ideally, in a true microscopic model, the full Hamiltonian should include the energetic contribution due to the confinement effect at high concentration; however, as the MaierSaupe theory employs a phenomenological pseudopotential, such concentration effects need to be reflected in the parameter $u_{q l}$. At high concentration, as the polymer chains form local layered structure between aligned mesogenic domains, both the side-group and the LMWLC mesogens experience the confinement effect due to the local smectic order, as illustrated in Figure 3c. In this regime, all mesogens experience similar enhancement of nematic order due to the confinement, and therefore, the cross-interaction parameter $u_{q l}$ should be approximately equal to $u_{q q}$. In the opposite limit at low concentration, as shown in Figure $3 \mathrm{~b}$, such confinement effects are no longer operative and the cross-interaction should be closer to $u_{l l}$. As the simplest approximation, we assume that $u_{q l}$ various linearly with the concentration as

$$
u_{q l}=u_{l l}+\phi\left(u_{q q}-u_{l l}\right)
$$

Although the variation of $u_{q l}$ with concentration may not be simply linear, this assumption serves as a first step in investigating the concentration-dependent confinement effect caused by the polymer backbone. We note that the form of $u_{q l}$ in eq 21 leads to a nematic-isotropic phase boundary with a negative azeotrope at low concentration and a positive azeotrope at high concentration; the appearance of azeotropes, or the concavity in the phase boundary, may be adjusted by modifying the functional dependence of $u_{q l}$ on $\phi$. Although the Scruggs-Kornfield experiment does not provide strong evidence for a negative azeotrope within experimental error, we adopt the functional form of $u_{q l}$ in eq 21 , as the possibility for an SCLCP/LMWLC mixture having a nematic-isotropic phase boundary with both the positive and the negative azeotropes may be of theoretical and experimental interest. For comparison, we have also calculated the phase diagram and the nematic order parameters using the BJL model. In that case, we use $u_{q l}=c\left(u_{q q} u_{l l}\right)^{1 / 2}$ for the cross-interaction parameter with $c=$ 1.2; the rest of the parameters remain the same.

The Flory-Huggins $\chi$ parameter characterizes the solvent quality. Although the dependence of $\chi$ on temperature could in principle be different in the nematic and the isotropic phases, ${ }^{29}$ the difference is usually ignored at the phenomenological level. ${ }^{21}$ (Note, however, the nematic ordering does alter the net interaction between the SCLCP and the LMWLC in our model due to the Maier-Saupe interactions.) In the ScruggsKornfield experiment, the chemical structure of the LMWLC mesogen is almost identical to the SCLCP side group, and thus we expect good miscibility between the SCLCP and the LMWLC. ${ }^{64,63}$ Indeed, the SCLCP was found to dissolve very well in the LMWLC, and the biphasic region in the phase diagram was observed to be very narrow. ${ }^{30}$ We thus use $A=0$ and a small negative value of $B=-0.5 / k_{B}$ for the $\chi$ parameter to ensure the good solvent quality. Since the SCLCP backbone chain in the Scruggs-Kornfield experiment is long, we set $N=$ 100 in our calculation. In the next two sections, we discuss the resulting phase behavior of the mixture and the nematic order of each component.

B. Phase Diagram. In this part, we discuss the phase diagram of the mixture. At intermediate concentrations, the volume fractions of the SCLCP side groups and the LMWLCs are comparable, and thus the cross-interaction between them is the most significant in this region. As the concentration of SCLCP increases beyond the overlap concentration, the SCLCPs start to tangle up, and thus, the confinement effects would increase the strength of cross-coupling, causing the nematic phase to be more favorable as $\phi$ increases. On the other hand, at both high and low concentrations, the behavior of the system is largely determined by self-interactions. In the limit of pure SCLCP, adding a small amount of LMWLC to the 
system increases the total concentration of mesogenic groups (because the mesogen density is higher in the pure LMWLC than in the pure SCLCP due to volume taken by the backbone and spacer in the latter). Therefore, we anticipate that the nematic phase becomes more favorable and $T_{\mathrm{NI}}$ increases with decreasing $\phi$ at the pure SCLCP end. In the opposite limit of pure LMWLC, adding a small amount of SCLCP reduces the concentration of mesogens and frustrates the liquid crystalline order due to local coupling effects, and $T_{\mathrm{NI}}$ therefore decreases with increasing $\phi$.

We show the phase diagram calculated using our model in Figure $4 \mathrm{a}$. The coexistence curves have a minimum at $T_{\mathrm{NI}}=27$

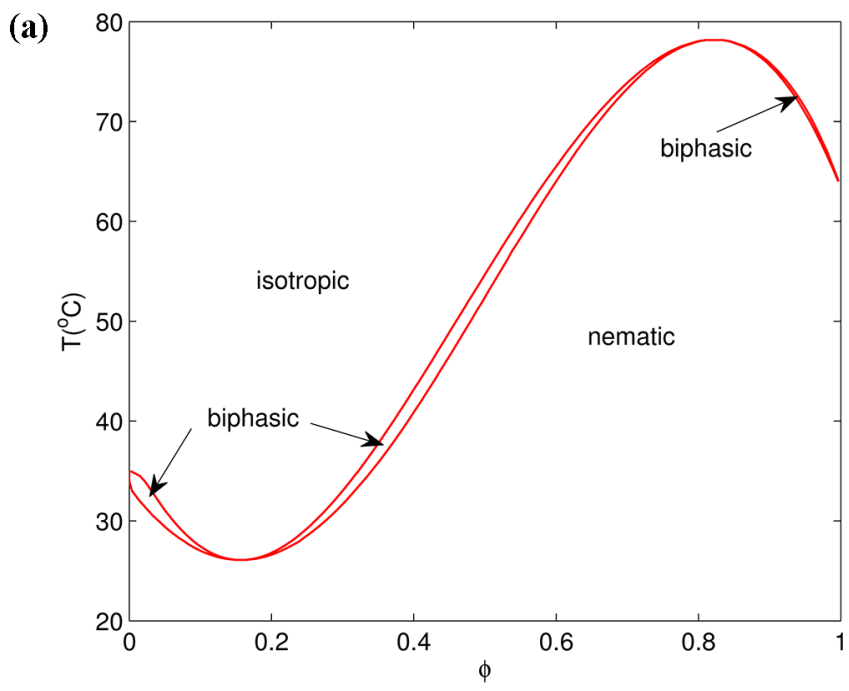

(b)

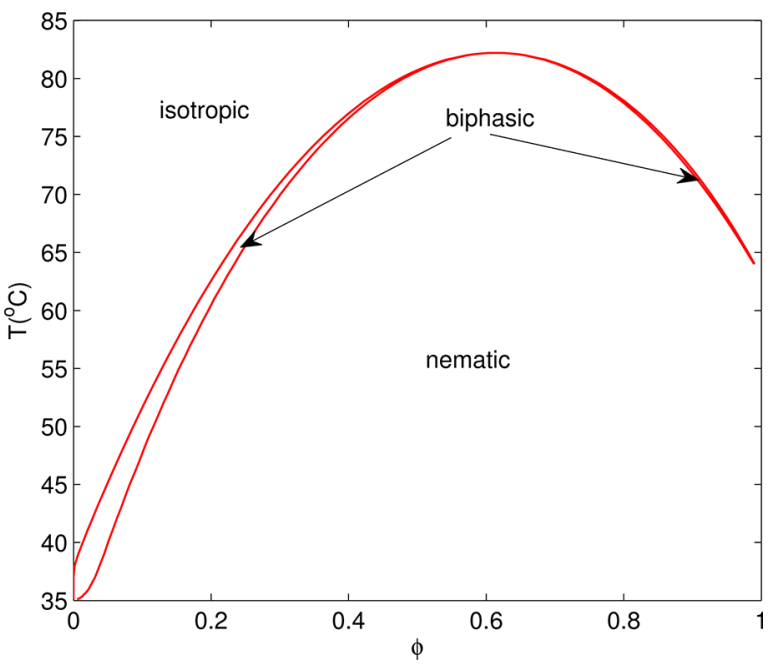

Figure 4. Phase diagram of the SCLCP/LMWLC mixture calculated using (a) our model, and (b) the BJL model.

${ }^{\circ} \mathrm{C}$ and $\phi=0.16$, and a maximum at $T_{\mathrm{NI}}=78^{\circ} \mathrm{C}$ and $\phi=0.82$, and the phase diagram is strongly asymmetric. The position of the maximum $T_{\mathrm{NI}}$ matches closely with the experimentally observed value of $T_{\mathrm{NI}}=78{ }^{\circ} \mathrm{C}$ at $\phi=0.78$. The nematicisotropic boundary is convex at low concentrations and concave at high concentrations. There is a narrow biphasic region between the coexistence curves, consistent with the good solvent quality of the mixture. The phase diagram calculated using the BJL model is shown in Figure $4 \mathrm{~b}$ for comparison. The result from the BJL model is qualitatively different from the result from our model. It predicts the maximum $T_{\mathrm{NI}}$ to occur at a much lower concentration of $\phi \approx 0.6$ and no minimum. The nematic-isotropic phase boundary is concave for all concentrations. However, while our model reproduces the main qualitative features of the experimentally observed phase diagram, (in which the phase boundary is convex at low concentration and concave at high concentration), it exaggerates the curvature of the phase boundary in the lowconcentration regime. A possible reason for this is that the increase in $u_{q l}$ with $\phi$ is faster than the simple linear relationship assumed in our calculation at low concentrations. The SCLCP in the Scruggs-Kornfield experiment has a high grafting density (which results in a higher persistence length in the polymer backbone due to steric factors) and a high degree of polymerization, which results in a large radius of gyration and a low overlap concentration. In a small angle neutron scattering experiment, Scruggs report that a solution with $5 \%$ weight of the same SCLCP in the nematic solvent is already in the semidilute regime. ${ }^{18}$ We thus expect that the confinement effect should set in at rather low concentrations, and $u_{q l}$ should increase rapidly with $\phi$ once the concentration exceeds the overlap concentration. To capture the behavior of the phase diagram more accurately, we should appropriately account for the nonlinear relationship between $u_{q l}$ and $\phi$.

C. Nematic Order Parameters. From our discussions in section II, it is clear that the BJL theory is unable to predict the relative magnitudes of the order parameters between the different components observed in the experiment: the order parameter of the LMWLC is greater than that of the SCLCP side-group, even though $T_{\mathrm{NI}}$ of the SCLCP is about $28{ }^{\circ} \mathrm{C}$ higher. ${ }^{30}$ The $\mathrm{BJL}$ model and its variants would predict that the component with a higher $\mathrm{T}_{\mathrm{NI}}$ always has a higher nematic order parameter. As alluded to earlier, a key ingredient missing in the BJL model is the coupling between the polymer backbone and the mesogens. Here by properly accounting for the local coupling between the backbone segments and the grafted side groups, as well as the global coupling between the backbone segments and the mesogenic groups, our theory is able to successfully capture the relative magnitudes of the nematic orders of the components. In addition, the trends in the variation of nematic orders with the concentration are also consistent with the experimental observation.

In parts $\mathrm{a}$ and $\mathrm{b}$ of Figure 5, we show the dependence of nematic order parameters on the concentration at $25{ }^{\circ} \mathrm{C}$ calculated using our model and the BJL model. The predictions from the two models are conspicuously different. On the basis of the results of our model, the nematic order parameters for both the side-group mesogens and the LMWLCs show a nonmonotonic dependence on the concentration. In the lowconcentration region of $\phi<0.15$, the addition of SCLCP destabilizes the nematic order of the LMWLC. This is because, first, the addition of SCLCP reduces the net concentration of mesogens in the system, and second, the perpendicular orientation of the polymer chain disturbs the ordering in the LMWLC. It is noteworthy that the SCLCP side-group has a lower nematic order parameter than the LMWLC at very small concentrations of $\phi<0.03$, as shown in the inset of Figure 5a. This behavior can be attributed to the fact that the local coupling interaction involves the SCLCP side groups directly, and therefore, its effect in suppressing the nematic order is greater on the side groups than that on the LMWLC mesogens. Furthermore, while the order parameter of the LMWLC decreases considerably as the concentration increases, the order 
(a)

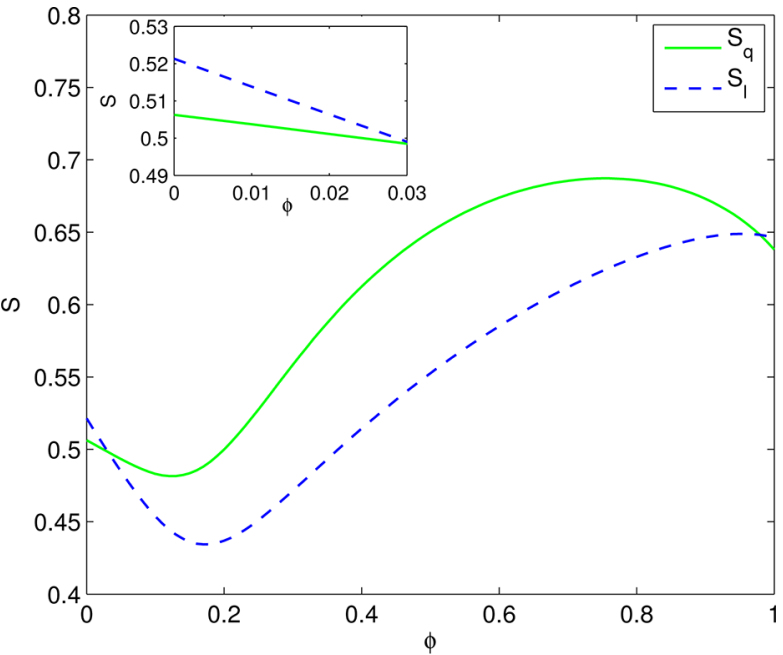

(b)

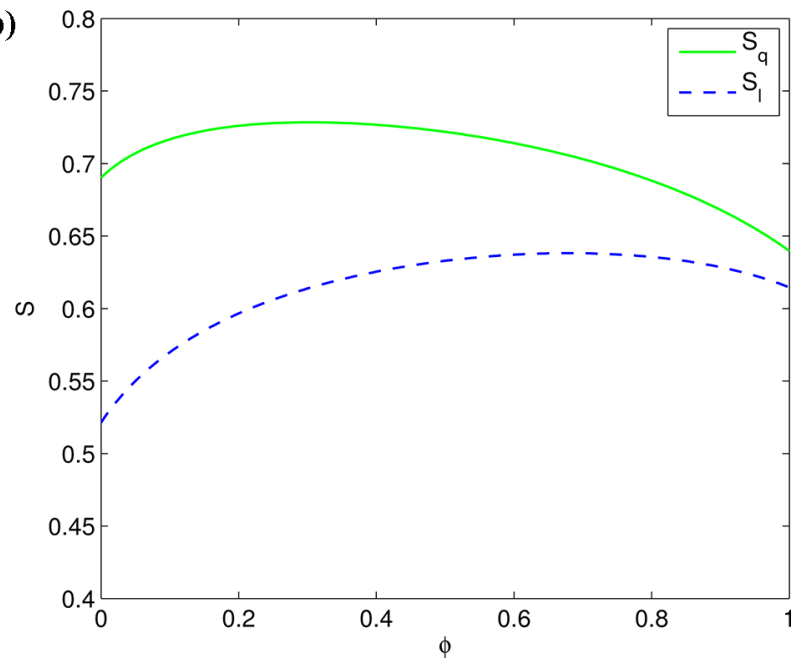

Figure 5. Nematic order parameters of the mesogenic groups at $25^{\circ} \mathrm{C}$ calculated using (a) our model, and (b) the BJL model.

of the SCLCP side-group is relatively insensitive to concentration. This behavior is due to the competition between global coupling and local coupling in the SCLCP, as the frustration in the SCLCP makes the nematic order of the SCLCP side-group less responsive to changes in the concentration. These calculated results are in excellent agreement with the experimental observation described in section II. $^{30}$ In the regime of intermediate to high concentration, the confinement effect on the nematic order becomes increasingly significant as $\phi$ increases due to the overlap of the polymer chains. As a result, the LMWLCs become increasingly ordered as the SCLCP concentration increases. The situation for the SCLCP side groups at high concentration is slightly more complicated, as $S_{q}$ first increases at intermediate $\phi$ due to the confinement effect, and then drops because of the decrease in mesogen concentration at very high $\phi$ values. In all concentration regimes, the presence of local couplings with the SCLCP backbone reduces the nematic order of the mesogenic groups, but the effect on the side-group mesogens is stronger due to the direct local coupling.

As a related phenomenon, in Figure 6 we show the temperature dependence of nematic order parameters at $\phi=$ 0.01 for temperatures below $T_{\mathrm{NI}}$ of the LMWLC. As expected, the nematic orders of both the SCLCP side groups and the

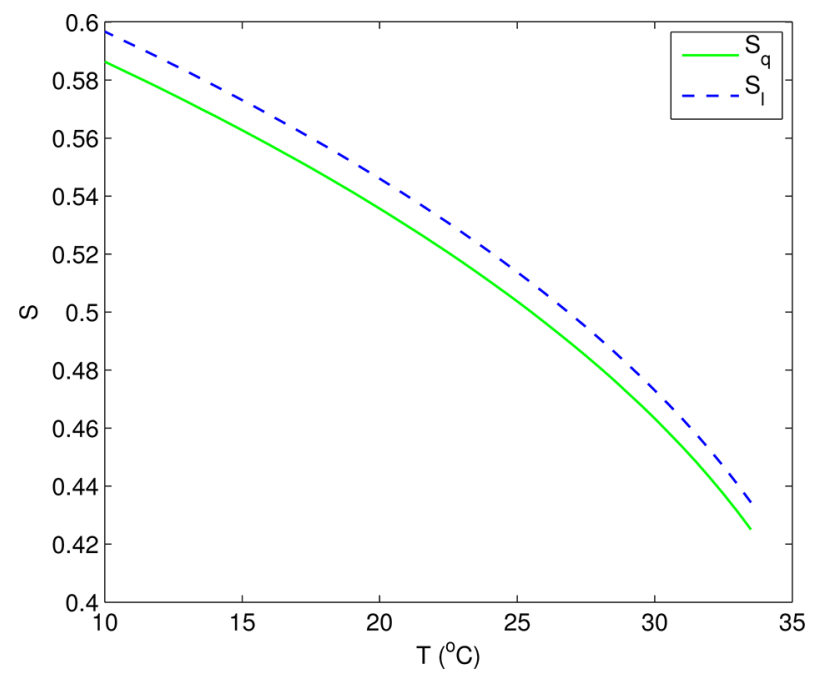

Figure 6. Nematic order parameters as a function of temperature at $\phi$ $=0.01$.

LMWLCs decrease with temperature. The more interesting observation is that the nematic order of the SCLCP side-group remains smaller than that of the LMWLCs, even though the pure SCLCP has a much higher $T_{\mathrm{NI}}$ than the pure LMWLC. This result is also in agreement with the experimental observation by Scruggs and Kornfield. ${ }^{30}$

D. Chain Conformation. The inclusion of nematic order of the SCLCP backbone segments not only provides more realistic description of the interactions in the system, but also gives rise to changes in the chain conformation. For the freely jointed chain model adopted here, the chain anisotropic ratio, $A$, is related to the nematic order parameter of the backbone segments by the following expression:

$$
A=\frac{R_{\|}}{R_{\perp}}=\sqrt{\frac{1+2 S_{p}}{1-S_{p}}}
$$

where $R_{\|}$and $R_{\perp}$ are the radius of gyration in the direction parallel and perpendicular to the nematic director, respectively.

In Figure 7, we show the behavior of chain anisotropic ratio as a function of concentration. For our choice of model

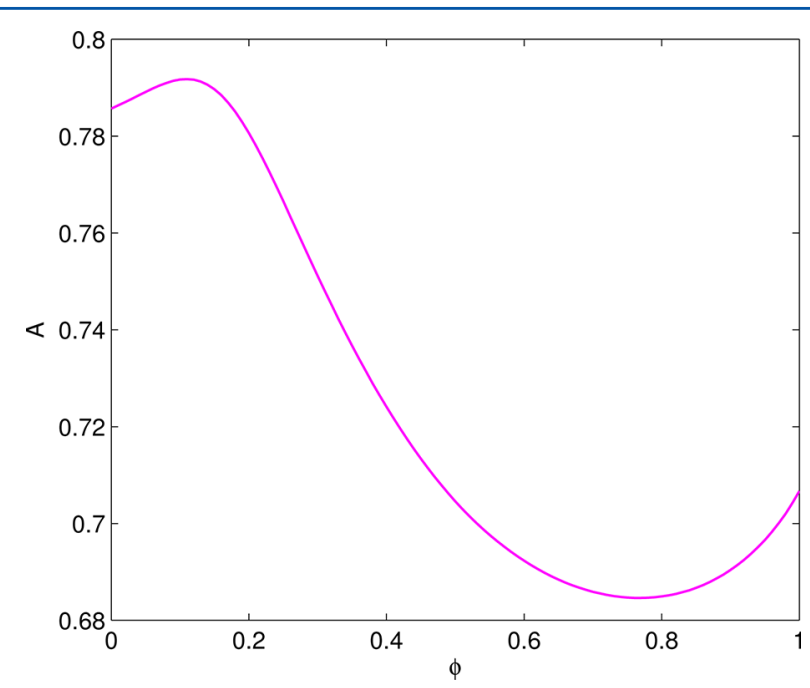

Figure 7. Dependence of chain anisotropic ratio on concentration at $T$ $=25{ }^{\circ} \mathrm{C}$. 
parameters, the order parameter $S_{p}<0$ in the entire concentration range because of the moderately strong end-on local coupling. Therefore, we have $A<1$, which means $R_{\|}<R_{\perp}$ and the polymer chain is in the shape of an oblate ellipsoid. This is in agreement with observations from small-angle neutron scattering experiments. ${ }^{52-56}$ Since the order parameter of the backbone has an opposite sign to the order parameter of the side-chain mesogens, the anisotropic ratio is negatively correlated with the nematic order parameter of the side-chain, $S_{q}$. The variation of $A$ with concentration can therefore be understood in light of the behavior of $S_{q}$ shown in Figure 5a. The anisotropic ratio changes nonmonotonically with concentration, such that the chain is the least oblate when $\phi \approx 0.16$, and most oblate when $\phi \approx 0.82$. However, if the local hinging is loose (i.e., the local coupling is weak), the shape of the polymer may become prolate due to the global coupling. ${ }^{31}$

\section{CONCLUSION}

In this paper, we have studied the phase behavior and the nematic order in SCLCP/LMWLC mixtures using a selfconsistent-field approach. By accounting for the local coupling and the confinement effect-both attributed to the presence of the polymer backbone-we have qualitatively captured the experimentally observed trend in the concentration-dependence of the transition temperature and the nematic order parameters. We find that the anomalous behavior in the temperature-composition phase diagram and the order parameter-composition dependence is mostly due to the effects of the polymer backbone. At low concentrations, the volume occupied by the polymer backbone reduces the overall mesogen concentration and weakens the nematic order, whereas at high concentrations, the restricted freedom of the mesogenic groups attached to the SCLCP backbone gives rise to local smectic fluctuations that reinforces the nematic order. The resulting phase diagram has a nematic-isotropic phase boundary that changes from convex to concave as concentration increases. In addition, as the competition between global and local couplings frustrates the ordering in the sidegroup mesogens, the side-group mesogens are shown to have lower nematic order than the LMWLCs at very low concentrations. Furthermore, our study predicts that the SCLCP adopts an oblate conformation in the mixture, but the oblateness can vary nonmonotonically with concentration.

In capturing the confinement effects caused by the local smectic fluctuations induced by the attachment of the mesogens to the SCLCP backbone, we have taken a phenomenological approach by expressing the nematic crossinteraction parameter $u_{q l}$ as a linear interpolation between $u_{q q}$ and $u_{l l}$ with concentration $\phi$. This crude approximation results in an exaggeration of the decrease in $T_{\mathrm{NI}}$ and the nematic order at low concentrations. As this system has a small overlap concentration due to the large degree of polymerization, we anticipate that $u_{q l}$ increases rapidly with $\phi$ at low concentration such that, when small amounts of SCLCP are added to the pure LMWLC, the effect of decreasing mesogen concentration can be counterbalanced by the increase in the strength of nematic interactions. The relationship between the global coupling parameters and the concentration of SCLCP is an interesting question for further studies; such relationship may also be altered, for example, with the use of ferroelectric nanoparticles. ${ }^{65,66}$ Furthermore, as local smectic fluctuations occur at high SCLCP concentration, the system may have a smectic phase at lower temperatures. Incorporating a possible smectic phase and exploring its consequences by combining our current theory with the McMillan theory is also an interesting future direction. ${ }^{67}$ Beyond the SCLCP and its mixtures, our work can also be readily extended to investigate the phase structure, the nematic order, and the conformation of MCSCLCPs in nematic solvents. ${ }^{68}$ Another extension of our current work is to explore mixtures of coil/liquid-crystalline diblock copolymers in nematic solvents. This may be done by including additional interaction terms due to the coil block in the copolymer and the block connectivity. These systems are known to exhibit a variety of equilibrium morphologies, and it will be interesting to investigate the interplay between microphase separation and the nematic ordering of the mesogenic groups. 69,70

\section{AUTHOR INFORMATION}

\section{Corresponding Author}

*E-mail: zgw@caltech.edu.

\section{Notes}

The authors declare no competing financial interest.

\section{ACKNOWLEDGMENTS}

We thank Rui Wang for helpful discussions. This work was supported by the NSF under Grant CHE-1040558, and B.Z. gratefully acknowledges support as an A-STAR fellow.

\section{REFERENCES}

(1) Ober, C. K.; Weiss, R. A. In Liquid-Crystalline Polymers; Weiss, R. A., Ober, C. K., Eds.; ACS Symposium Series 435; American Chemical Society: Washington, DC, 1990; Chapter 1, pp 1-13.

(2) Platé, N. A., Ed. Liquid-Crystal Polymers; Plenum Press: New York, 1993.

(3) Wang, X. J.; Zhou, Q. F. Liquid crystalline polymers; World Scientific: Singapore, 2004.

(4) Donald, A. M.; Windle, A. H.; Hanna, S. Liquid Crystalline Polymers, 2nd ed.; Cambridge University Press: Cambridge, U.K., 2006.

(5) Shibaev, V. P. J. Polym. Sci., Ser. A 2009, 51, 1131-1193.

(6) Blumstein, A.; Thomas, O. Macromolecules 1982, 15, 1264-1267.

(7) Finkelmann, H.; Kock, H. J.; Rehage, G. Makromol. Chem., Rapid Commun. 1981, 2, 317-322.

(8) Reck, B.; Ringsdorf, H. Makromol. Chem., Rapid Commun. 1985, 6, 291-299.

(9) Ringsdorf, H.; Schneller, A. Br. Polym. J. 1981, 13, 43-46.

(10) Hessel, F.; Finkelmann, H. Polym. Bull. 1985, 14, 375-378.

(11) Trimmel, G.; Riegler, S.; Fuchs, G.; Slugovc, C.; Stelzer, F. In Metathesis Polymerization; Buchmeiser, M., Ed.; Advances in Polymer Science; Springer: Nethelands, 2005; Vol. 176; pp 43-87.

(12) Mattoussi, H.; Ober, R. Macromolecules 1990, 23, 1809-1816.

(13) Yao, N.; Jamieson, A. M. Macromolecules 1997, 30, 5822-5831.

(14) Chiang, Y.-C.; Jamieson, A. M.; Zhao, Y.; Kasko, A. M.; Pugh, C. Polymer 2002, 43, 4887-4894.

(15) Mattoussi, H.; Ober, R.; Veyssie, M.; Finkelmann, H. Europhys. Lett. 1986, 2, 233.

(16) Kempe, M. D.; Kornfield, J. A.; Lal, J. Macromolecules 2004, 37, $8730-8738$.

(17) Brochard, F. J. Polym. Sci., Polym. Phys. Ed. 1979, 17, 13671374.

(18) Scruggs, N. R. Coupling polymer thermodynamics and viscoelasticity to liquid crystalline order: self-assembly and relaxation dynamics of block copolymers in a nematic solvent. Ph.D. thesis, California Institute of Technology: Pasadena, CA, 2007.

(19) Finkelmann, H.; Rehage, G. In Liquid Crystal Polymers II/III; Gordon, M., Platé, N., Eds.; Advances in Polymer Science 99-172; Springer-Verlag: Berlin, 1984; Vol. 60-61; pp 99-172.

(20) Brochard, F.; Jouffroy, J.; Levinson, P. J. Phys. (Paris) 1984, 45, $1125-1136$. 
(21) Chiu, H.-W.; Kyu, T. J. Chem. Phys. 1995, 103, 7471-7481.

(22) Chiu, H.-W.; Zhou, Z. L.; Kyu, T.; Cada, L. G.; Chien, L. C. Macromolecules 1996, 29, 1051-1058.

(23) Flory, P. J. Principles of Polymer Chemistry; Cornell University: Ithaca, NY, 1953.

(24) Rubinstein, M.; Colby, R. H. Polymer Physics; Oxford University Press: Oxford, U.K., 2009.

(25) Maier, W.; Saupe, A. Z. Naturforsch. 1959, 14, 882-889.

(26) Maier, W.; Saupe, A. Z. Naturforsch. 1960, 15, 287-292.

(27) de Gennes, P. G.; Prost, J. The Physics of Liquid Crystals, 2nd ed.; Oxford University Press: Oxford. U.K., 1993.

(28) Casagrande, C.; Veyssié, M.; Finkelmann, H. J. Phys. (Paris) 1982, 43, 671-675.

(29) Sigaud, G.; Achard, M. F.; Hardouin, F.; Coulon, C.; Richard, H.; Mauzac, M. Macromolecules 1990, 23, 5020-5024.

(30) Scruggs, N. R.; Kornfield, J. A. Macromol. Chem. Phys. 2007, 208, 2242-2253.

(31) Wang, R.; Wang, Z.-G. Macromolecules 2010, 43, 10096-10106.

(32) Wang, X. J.; Warner, M. J. Phys. A 1987, 20, 713-731.

(33) Wessels, P. P. F.; Mulder, B. M. Europhys. Lett. 2003, 64, 337343.

(34) Shibaev, V. P.; Platé, N. A. In Liquid Crystal Polymers II/III; Gordon, M., Platé, N. A., Eds.; Advances in Polymer Science 173-252; Springer-Verlag: Berlin, 1984; Vol. 60-61; pp 173-252.

(35) Stevens, H.; Rehage, G.; Finkelmann, H. Macromolecules 1984, 17, 851-856.

(36) Maughon, B. R.; Weck, M.; Mohr, B.; Grubbs, R. H. Macromolecules 1997, 30, 257-265.

(37) Zhao, M.; Bautista, M.; Ford, W. T. Macromolecules 1991, 24, 844-849.

(38) Craig, A. A.; Imrie, C. T. Macromolecules 1999, 32, 6215-6220.

(39) Boiko, N.; Shibaev, V.; Ostrovskii, B.; Sulyanov, S.; Wolff, D.; Springer, J. Macromol. Chem. Phys. 2001, 202, 297-303.

(40) Kihara, H.; Kishi, R.; Miura, T.; Kato, T.; Ichijo, H. Polymer 2001, 42, 1177-1182.

(41) Pereira, F. V.; Zandoná, R.; Borsali, R.; Merlo, A. A.; da Silveira, N. P. Macromol. Symp. 2005, 229, 93-98.

(42) Spakowitz, A. J.; Wang, Z.-G. J. Chem. Phys. 2003, 119, 1311313128.

(43) Fredrickson, G. H. The Equilibrium Theory of Inhomogeneous Polymers; Oxford University Press: Oxford, U.K., 2006.

(44) Olsen, B. D.; Jang, S. Y.; Lüning, J. M.; Segalman, R. A. Macromolecules 2006, 39, 4469-4479.

(45) Zhao, Y.; Jamieson, A. M.; Olson, B. G.; Yao, N.; Dong, S.; Nazarenko, S.; Hu, X.; Lal, J. J. Polym. Sci. Polym. Phys. 2006, 44, 2412-2424.

(46) Rousseau, D.; Marty, J. D.; Mauzac, M.; Martinoty, P.; Brandt, A.; Guenet, J. M. Polymer 2003, 44, 2049-2055.

(47) Sluckin, T. J.; Shukla, P. J. Phys. A 1983, 16, 1539-1553.

(48) Dubault, A.; Ober, R.; Veyssie, M.; Cabane, B. J. Phys. (Paris)

1985, 46, 1227-1232.

(49) Matsuyama, A.; Kato, T. Phys. Rev. E 1998, 58, 585.

(50) Matsuyama, A.; Kato, T. J. Chem. Phys. 1998, 109, 2023.

(51) Matsuyama, A.; Kato, T. Phys. Rev. E 1999, 59, 763.

(52) Kirste, R. G.; Ohm, H. G. Makromol. Chem., Rapid Commun. 1985, 6, 179-185.

(53) Noirez, L.; Cotton, J. P.; Hardouin, F.; Keller, P.; Moussa, F.; Pepy, G.; Strazielle, C. Macromolecules 1988, 21, 2889-2891.

(54) Noirez, L.; Keller, P.; Davidson, P.; Hardouin, F.; Cotton, J. P. J. Phys. (Paris) 1988, 49, 1993-1999.

(55) Noirez, L.; Keller, P.; Cotton, J. P. Liq. Cryst. 1995, 18, 129148.

(56) Cotton, J. P.; Hardouin, F. Prog. Polym. Sci. 1997, 22, 795-828.

(57) Fourmaux-Demange, V.; Boué, F.; Brûlet, A.; Keller, P.; Cotton, J. P. Macromolecules 1998, 31, 801-806.

(58) Brûlet, A.; Fourmaux-Demange, V.; Cotton, J. P. Macromolecules 2001, 34, 3077-3080.

(59) Renz, W.; Warner, M. Phys. Rev. Lett. 1986, 56, 1268-1271.

(60) Rieger, J. J. Phys. (Paris) 1988, 49, 1615-1625.
(61) Benmouna, F.; Maschke, U.; Coqueret, X.; Benmouna, M. Polym. Int. 2001, 50, 469-474.

(62) Kim, N.; Choi, J.; Chien, L. C.; Kyu, T. Macromolecules 2007, 40, 9582-9589.

(63) Benmouna, R.; Benmouna, M. J. Chem. Eng. Data 2010, 55, $1759-1767$.

(64) Kempe, M. D.; Kornfield, J. A.; Ober, C. K.; Smith, S. D. Macromolecules 2004, 37, 3569-3575.

(65) Lopatina, L. M.; Selinger, J. V. Phys. Rev. Lett. 2009, 102, 197802.

(66) Lopatina, L. M.; Selinger, J. V. Phys. Rev. E 2011, 84, 041703.

(67) Kyu, T.; Chiu, H.-W.; Kajiyama, T. Phys. Rev. E 1997, 55, 71057110.

(68) Yang, G.; Tang, P.; Yang, Y. Macromolecules 2012, 45, 35903603.

(69) Shah, M.; Pryamitsyn, V.; Ganesan, V. Macromolecules 2008, 41, 218-229.

(70) Scruggs, N. R.; Verduzco, R.; Uhrig, D.; Khan, W.; Park, S. Y.; Lal, J.; Kornfield, J. A. Macromolecules 2008, 42, 299-307. 